\title{
Composición, diversidad y estructura de un bosque manejado del centro de México
}

\author{
Composition, diversity, and structure of a managed forest in central \\ Mexico
}

\author{
Martha Azucena Rendón-Pérez', Patricia Hernández-de la Rosal*, \\ Alejandro Velázquez-Martínez', José Luis Alcántara-Carbajal² y Valentín José Reyes-Hernández'
}

\begin{abstract}
1 Colegio de Postgraduados. Campus Montecillo. Postgrado en Ciencias Forestales. Montecillo, Estado de México, México.
\end{abstract}

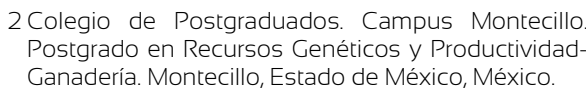

* Autor de correspondencia. pathr@colpos.mx .

\section{RESUMEN}

La caracterización estructural de la vegetación facilita la toma de decisiones de manejo forestal y la prescripción de prácticas silvícolas adecuadas a los propósitos del propietario. En el ejido Nopalillo, municipio de Epazoyucan, al sureste de Hidalgo, México, se realiza aprovechamiento forestal maderable en tres asociaciones arbóreas, cada una dominada por Pinus montezumae Lamb., Pinus pseudostrobus Lindl. y Pinus patula Schltdl. et Cham., respectivamente; además, el ejido cuenta con una superficie dominada por Pinus-Quercus que ha sido destinada a la conservación. Con el objetivo de valorar composición, diversidad y estructura de estas asociaciones, se analizó información de los estratos arbóreo, arbustivo y herbáceo de 79 sitios de muestreo. Se calcularon índices de valor de importancia, de diversidad de Margalef, de Simpson y de Sorensen y se describen las estructuras vertical y horizontal de la vegetación. La asociación Pinus-Quercus presentó la mayor área basal $\left(28 \mathrm{~m}^{2} / \mathrm{ha}\right)$, diámetro normal y altura promedio $(20.5 \mathrm{~cm}$ y $10.7 \mathrm{~m}$, respectivamente), la mayor proporción de árboles de dimensiones grandes (diámetro normal $>60 \mathrm{~cm}$ ) y una composición de especies diferente a las otras asociaciones. La mayor riqueza de especies se registró en las áreas bajo aprovechamiento maderable, pero la diversidad arbórea fue superior en el bosque de Pinus-Quercus. Estos resultados se proponen como referentes para la identificación de los efectos de las prácticas silvícolas sobre la vegetación y para el desarrollo de estrategias de manejo, conservación y uso del bosque.

PALABRAS CLAVE: aprovechamiento forestal maderable, arbolado, arbustos, hierbas, índice de valor de importancia, prácticas silvícolas.

\section{ABSTRACT}

Structural characterization of the vegetation facilitates forest management decision-making and prescription of silvicultural practices appropriate to the landowner's purposes. In the ejido Nopalillo, municipality of Epazoyucan, located southeast of Hidalgo, Mexico, forest harvest is practiced in three associations dominated by Pinus montezumae Lamb., Pinus psendostrobus Lindl., and Pinus patula Schl. et Cham., respectively. In addition, the ejido has an area dominated by Pinus-Quercus used as a conservation area. To characterize these four conditions, information from 79 sampling plots was analyzed, in which the arboreal, shrub and herbaceous strata were evaluated. Structural analysis of the vegetation was based on the Importance Value Index, Margalef, Simpson and Sorensen diversity indexes, as well as the vertical and horizontal structure in each association. The Pinus-Quercus area presented the largest basal area $\left(28 \mathrm{~m}^{2} / \mathrm{ha}\right)$, normalized diameter $(\mathrm{DBH})$ and average height $(20.5 \mathrm{~cm}$ and $10.7 \mathrm{~m})$, respectively, the highest proportion of large trees $(\mathrm{DBH}>60 \mathrm{~cm})$ and species composition different from the other associations. The greatest total diversity was found in the areas under timber harvesting, and the highest tree diversity in the conservation area. The results can be used as a reference to identify the effect of silvicultural practices, they are also the basis for the development of strategies for forest management, conservation, and use.

KEYWORDS: forest harvesting, trees, shrubs, herbs, importance value index, silvicultural practices. 


\section{INTRODUCCIÓN}

El manejo forestal sustentable (MFS) tiene como objetivo principal la producción maderable, además de asegurar la provisión de otros bienes y servicios. Entre los muchos atributos que interesa conocer de los bosques bajo manejo se encuentran la estructura, composición y diversidad de la vegetación, porque su conocimiento permite entender la condición actual del ecosistema y a partir de este, contar con información adecuada para prescribir prácticas silvícolas apropiadas.

La estructura del bosque puede definirse como la forma en que las plantas se organizan en el espacio (Kimmins, 1997) y considera tanto la distribución como la variación en las dimensiones de los individuos (Gadow y Hui, 1999; Franklin et al., 2002). La distribución está determinada por las estrategias de regeneración de las especies, la intervención silvícola y la fase de desarrollo del rodal. La variación de las dimensiones, por otra parte, se refiere a la relación de tamaño de los individuos que la componen y puede evaluarse, principalmente, a través de la medición de diámetros y alturas (Del Río, Montes, Cañelas y Montero, 2003).

Las investigaciones realizadas sobre la estructura de bosques manejados coinciden en que esta característica es un indicador de la diversidad de un bosque (Corral Rivas, Aguirre Calderón, Jiménez Pérez y Corral Rivas, 2005; Del Río et al., 2003) y que, a través de las intervenciones silvícolas, esta puede ser modificada. Por lo tanto, el conocimiento, el estudio, la evaluación y el monitoreo de la estructura son fundamentales para garantizar la sostenibilidad de los ecosistemas a través de la gestión y conservación (Castellanos-Bolaños et al., 2008).

En México se han desarrollado trabajos que han relacionado las prácticas silvícolas con la composición, diversidad y estructura de bosques templados de pino y pino-encino bajo manejo, particularmente en Chiapas, Durango, Jalisco, Nuevo León, Oaxaca y Puebla, pero considerando principalmente al estrato arbóreo (Solís Moreno, et al., 2006; Castellanos-Bolaños et al., 2008; Návar-Chaidez y González-Elizondo, 2009; HernándezSalas et al., 2013; Márquez-Linares, González-Elizondo y
Álvarez-Zagoya, 1999; Delgado Zamora et al., 2017; Graciano-Ávila et al., 2017; López-Hernández et al., 2017; Medrano Meraz, Hernández, Corral Rivas y Nájera Luna, 2017; Ramírez-Santiago et al., 2019; Monarrez-Gonzalez, Gonzalez-Elizondo, Marquez-Linares, Gutierez-Yurrita y Perez-Verdin, 2020).

Al comparar bosques con y sin manejo, en la mayoría de estas investigaciones se observaron cambios en riqueza, diversidad y composición florística y evidencia de variación en función del tiempo de aplicación de los tratamientos, tipo de corta e intensidad. Por otra parte, aquellos que consideran otras forma de vida como los arbustos y las hierbas (Luna-Bautista, Hernández-de la Rosa, VelázquezMartínez, Gómez-Guerrero y Acosta-Mireles, 2015; Vázquez-Cortez, Clark-Tapia, Manzano-Méndez, González-Adame y Aguirre-Hidalgo, 2018) o las epífitas (Wolf, 2005; Jiménez, Damoa, Ochoa-Gaona y ClarkTapia, 2014) son escasos; por ende, es importante realizar ese tipo de estudios en bosques manejados para poder relacionar la importancia de estos grupos taxonómicos con los diversos servicios ecosistémicos, como conservación del suelo, infiltración y flujo de agua, entre otros (MonárrezGonzález, Pérez-Verdín, López-González, MárquezLinares y González-Elizondo, 2018).

El ejido El Nopalillo, Hidalgo, cuenta con un bosque secundario, en donde se ha realizado aprovechamiento forestal por más de 100 años, inicialmente con cortas de selección a través de concesión a la industria forestal. Desde 1980, lleva a cabo un programa de manejo forestal que aplica el Método de Desarrollo Silvícola (MDS). El área cuenta con la certificación nacional de Manejo Forestal Sustentable (NMX-AA-143-SCFI-2015) y del Consejo de Administración Forestal (FSC, por sus siglas en inglés); sin embargo, no existe una caracterización estructural detallada sobre el efecto del manejo forestal en la vegetación o alguna evaluación que pudiera ser útil para guiar la prescripción de prácticas silvícolas. Se parte del supuesto de que existen diferencias en estructura, diversidad y composición entre las asociaciones sujetas a aprovechamiento maderable y aquella que no lo está, siendo las primeras menos complejas y diversas que la segunda. 


\section{OBJETIVOS}

Evaluar estructura, composición taxonómica y diversidad de especies del bosque templado del ejido Nopalillo, considerando las diferentes asociaciones vegetales presentes y sus estratos, además de relacionar el efecto de las prácticas silvícolas sobre los bosques manejados.

\section{MATERIALES Y MÉTODOS}

\section{Área de estudio y diseño de muestreo}

Se localiza en el Cerro de Las Navajas, Sierra de Pachuca, municipio de Epazoyucan, al sureste del estado de Hidalgo y forma parte del Eje Neovolcánico (Fig. 1). Comprende una zona serrana que presenta un gradiente altitudinal de
2800 m a 3100 m; con suelos de tipo Feozem y, en menor proporción, de tipo Andosol; el clima es semifrío subhúmedo y templado subhúmedo; se encuentra dentro de la cuenca del río Pánuco y los tipos de vegetación predominantes son el bosque de pino, seguido del de encino (Comisión Nacional Forestal [Conafor], 2014).

A partir de la información disponible en el programa de manejo (Asociación de Silvicultores de la Región Forestal Pachuca-Tualancingo, A.C., 2015), se identificaron 245 ha bajo aprovechamiento maderable que fueron clasificadas de la siguiente manera: Pinus montezumae Lamb. (en adelante Pmn), P. patula Schltdl. \& Cham. (Ppt) y P. pseudostrobus Lindl. (Pps) y las de conservación (61 ha) con asociación de Pinus-Quercus (PQ).
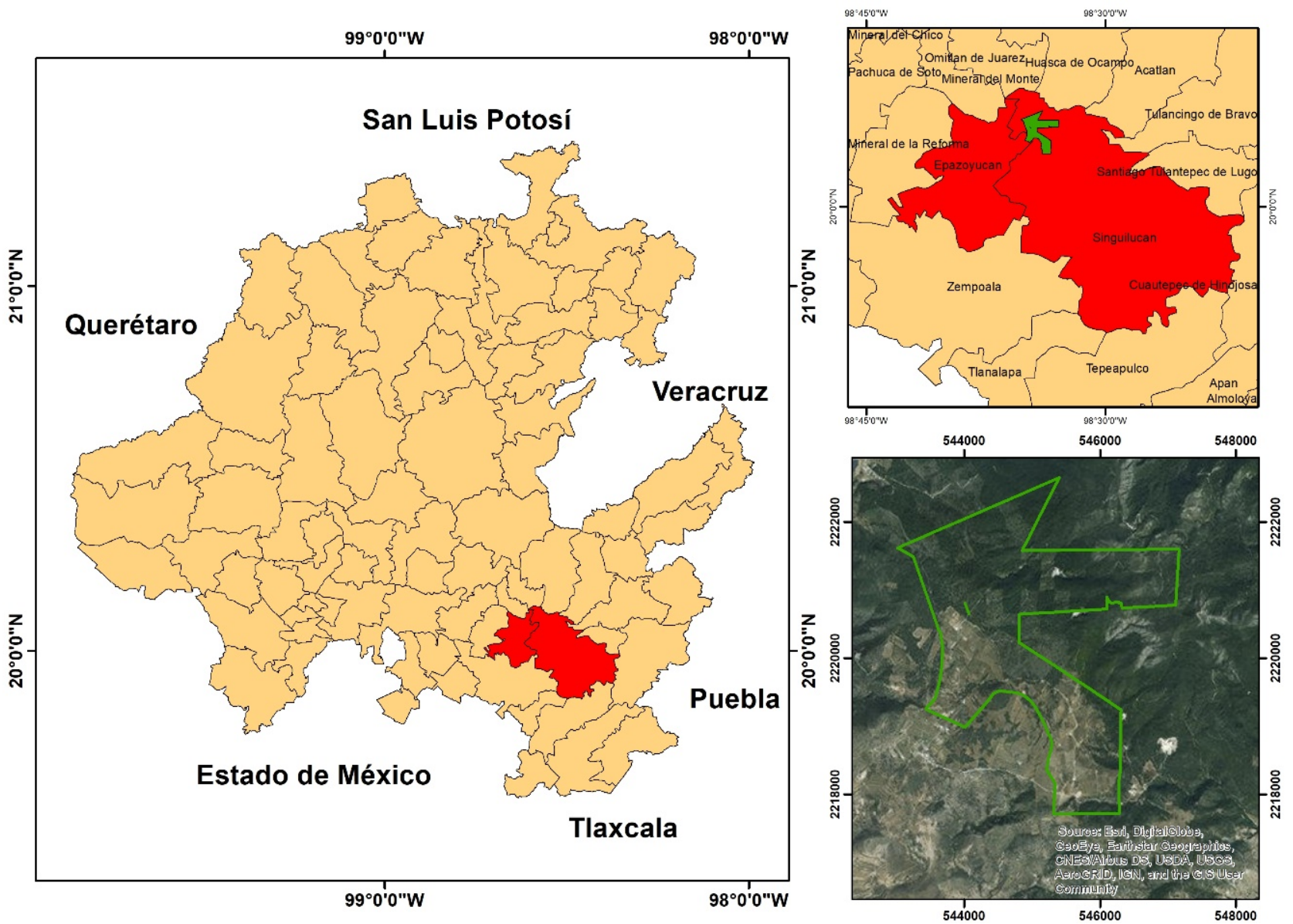

FiguRA 1. Localización del área de estudio, ejido Nopalillo, Epazoyucan, Hidalgo, México 
Se aplicó un diseño de muestreo sistemático estratificado (con intensidad de 1\%) (Wan Razali y Wan Mohd.Shukri, 1999), en el que los estratos correspondieron a cada una de las asociaciones vegetales y los puntos de muestreo se ubicaron a partir de sobreponer una malla con equidistancia entre puntos de $100 \mathrm{~m}$ en el área de estudio y elegir aleatoriamente los sitios para muestreo. Se seleccionaron 65 sitios en las asociaciones bajo manejo y 14 en el área de conservación.

El muestreo se llevó a cabo de junio a agosto de 2018; en cada sitio se registraron coordenadas geográficas, altitud, pendiente, exposición, profundidad de hojarasca, porcentaje de cobertura de piedras y tipo de asociación vegetal.

Las unidades de muestreo fueron sitios circulares con una área de $400 \mathrm{~m}^{2}$; de manera anidada se ubicó, en el centro, un subsitio circular de $12.56 \mathrm{~m}^{2}$ y cuatro subsitios cuadrados de $1 \mathrm{~m}^{2}$ localizados a $5 \mathrm{~m}$, a partir del centro del sitio, en dirección hacia cada punto cardinal (N, E, S y O) (Fig. 2).

En el sitio principal se evaluó el estrato arbóreo (individuos con diámetro normal $[\mathrm{DN}] \geq 7.5 \mathrm{~cm}$, medido a $1.30 \mathrm{~m})$, considerando las variables de DN (cm), altura total (At, $\mathrm{m}$ ), diámetro de copa en las orientaciones N-S y E-O (proyección sobre el suelo, m) y nombre científico. En el subsitio circular se midió el estrato arbustivo (individuos que ramificaban desde la base y con altura mayor a $25 \mathrm{~cm}$ ), se determinó la especie, la altura total (m), el diámetro de copa en las orientaciones N-S y E-O, el número de tallos y el diámetro basal promedio (cm). Finalmente, en los sitios cuadrados se registraron todas las especies del estrato herbáceo y su cobertura, además de la frecuencia en el caso de hierbas individuales (Conafor, 2017; Luna-Bautista et al., 2015).

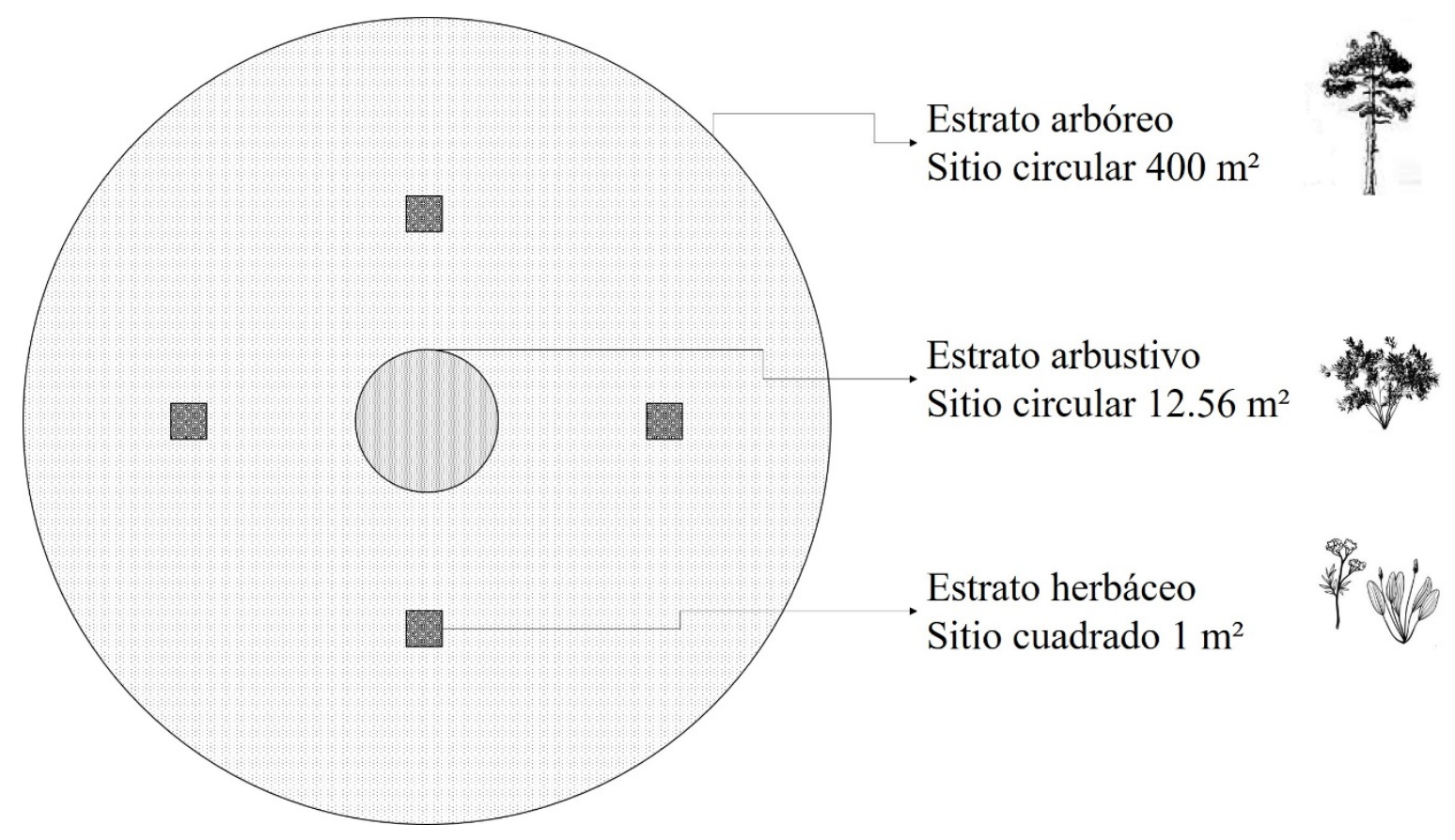

FIGURA 2. Esquema anidado de la unidad de muestreo (UM) para evaluación de vegetación. 
La determinación taxonómica se realizó en campo cuando se conocía la especie y en herbario a partir de colectas botánicas; se utilizaron las claves dicotómicas de la Flora Fanerogámica del Valle de México (Calderón de Rzedowski y Rzedowski, 2005), de la Flora del Bajío y de Regiones Adyacentes (González Elizondo y González Elizondo, 2014) y de la Flora de Veracruz (Nash y Nee, 1984). La nomenclatura científica se corroboró de acuerdo con la lista de nombres botánicos del portal de The Plant List (The Plant List, 2013).

La determinación de especies arbóreas se realizó en el herbario CHAP de la División de Ciencias Forestales de la Universidad Autónoma Chapingo; las hierbas de la familia Asteraceae en el herbario de la Preparatoria Agrícola de la misma universidad y el resto de las muestras del estrato herbáceo y arbustivo en el herbario CHAPA del Colegio de Postgraduados. Se constituyó el listado florístico incluyendo familia, género, nombre específico y autoridad taxonómica de cada especie.

\section{Análisis de la información}

Las estructuras horizontal y vertical del arbolado se representaron con histogramas generados a partir de la distribución de frecuencias de doce clases diamétricas de 5 $\mathrm{cm}$ de amplitud, desde $7.5 \mathrm{~cm}$ hasta más de $62.5 \mathrm{~cm}$ y la distribución de frecuencias de seis clases de altura de $5 \mathrm{~m}$, desde $0 \mathrm{~m}$ hasta $30 \mathrm{~m}$ (Luna-Bautista et al., 2015). El índice de valor de importancia relativa (IVI) se calculó para conocer la importancia ecológica en los diferentes estratos (arbóreo, arbustivo y herbáceo) para cada asociación vegetal con la fórmula: IVI $(\%)=1 / 3 \quad(\mathrm{AB}$ relativa + densidad relativa + frecuencia relativa) (Mueller-Dombois y Ellenberg, 1974).

Se calculó el índice de riqueza de Margalef con $R=$ $(S-1) / \ln N$ y de diversidad de Simpson (1-D) por estrato para cada asociación, a través de la fórmula

$$
D=\sum_{i=1}^{n} n i(n i-1) /((N(N-1))
$$

de acuerdo con Magurran (2004).
Para detectar diferencias de diversidad entre estratos dentro de las asociaciones (índice de Simpson e índice de Margalef), primero se verificó la normalidad de los datos a través de la prueba de Kolmogorov-Smirnov (Zar, 2010); debido a que estos no cumplieron los supuestos de normalidad y homocedasticidad, se aplicó la prueba no paramétrica de Kruskal-Wallis (Magurran, 2004), bajo la hipótesis nula $\mathrm{Ho}=$ hay igualdad de medias, con nivel de significancia de 5\% y utilizando el software R (The R Core Team, 2019).

Se estimó la similitud entre las cuatro asociaciones a través del índice de Sorensen (Cs)

$\left.C_{S}(\%)=\left(\frac{2 j}{(a+b)}\right) \times 100\right]$ (Magurran, 2004),

utilizando una hoja de cálculo en MS Excel ${ }^{\circledR}$. Este coeficiente es una medida inversa de la diversidad beta; cuando no hay especies compartidas el valor es 0 y cuando la composición es la misma el valor es 1 (Koleff, Gaston y Lennon, 2003).

\section{REsultados}

\section{Composición, riqueza y diversidad}

Se evaluaron 1752 árboles, 255 arbustos y 578 hierbas que correspondieron a 88 especies pertenecientes a 47 géneros y 29 familias (Anexo 1). Los géneros Pinus y Quercus tuvieron el mayor número de especies, mientras que las familias Pinacea y Asteraceae fueron las mejor representadas.

La composición florística en el estrato arbóreo estuvo integrada por 20 especies de 10 géneros y 8 familias. Pinus fue el género dominante, ya que representó $80 \%$ de los individuos muestreados, seguido de Quercus (10\%), mientras que las especies dominantes fueron Pinus montezumae y Pinus pseudostrobus (Fig. 3).

En el estrato arbustivo se registraron 10 especies de 10 géneros y 6 familias. Baccharis conferta Kunth y Ageratina glabrata Kunth fueron las especies dominantes en este caso (Fig. 4). 


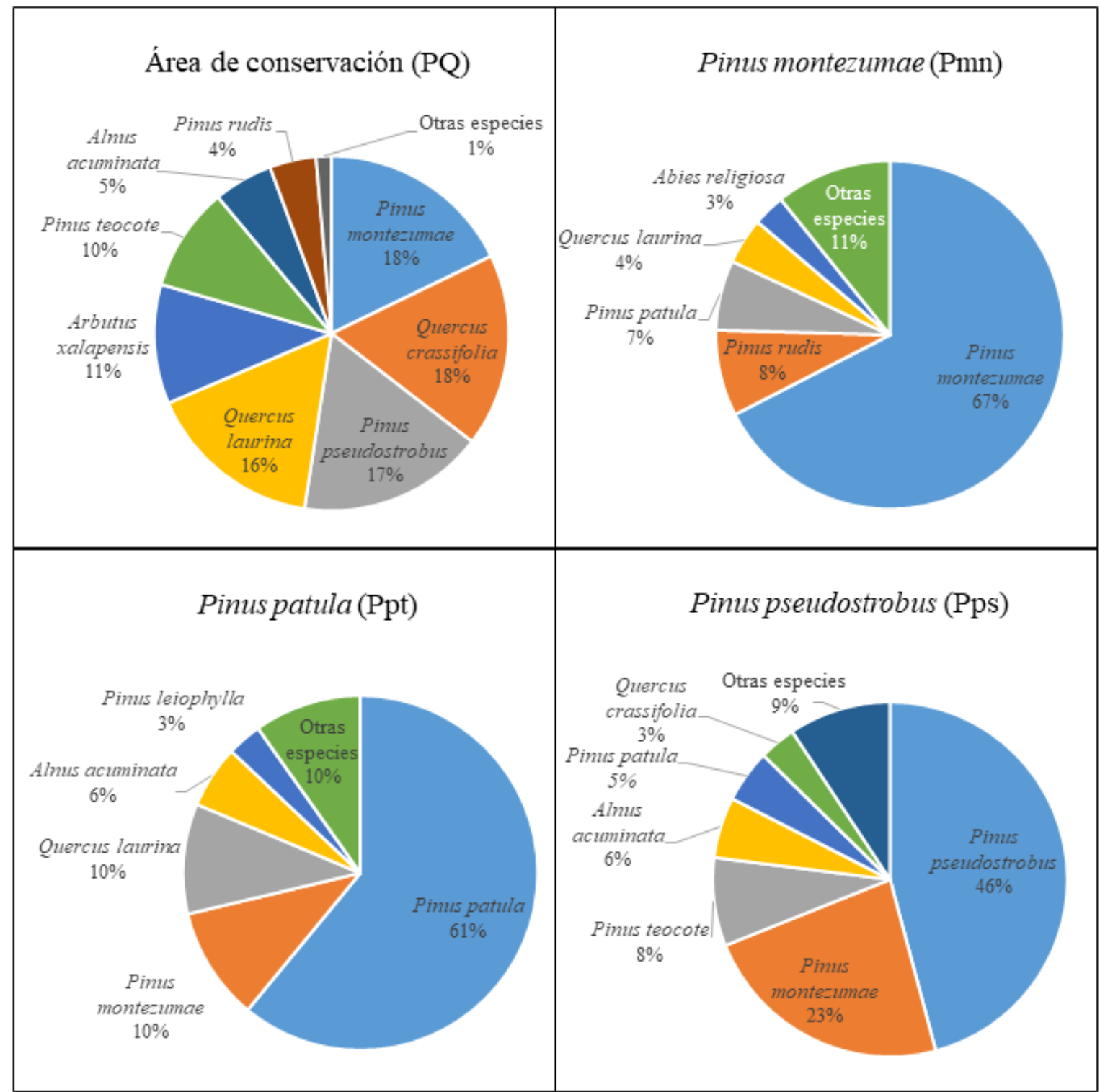

FIGURA 3. Porcentaje de importancia relativa por especie y asociación vegetal en el estrato arbóreo del ejido Nopalillo, Hidalgo, México.

En el estrato herbáceo se registraron 58 especies de 26 géneros y 24 familias. La familia mejor representada fue la Asteraceae, aunque las especies dominantes fueron Mublenbergia macroura (Humb., Bonpl. \& Kunth) Hitchc. y Lachemilla procumbens (Rose) Rydb (Fig. 5.).

Litsea glaucescens Kunth y Cupressus lusitanica Mill. fueron las únicas especies que se encontraron en el área de estudio y que han sido clasificadas dentro de alguna de las categorías de riesgo de la norma oficial mexicana NOM-059-
SEMARNAT-2010 de la Secretaría del Medio Ambiente y Recursos Naturales [Semarnat] (2010).

Se detectó variación en los índices de riqueza y de diversidad entre los estratos arbóreo, arbustivo y herbáceo, dentro de cada asociación (Tabla 1). Los índices de riqueza (Margalef) y de diversidad (Simpson) obtuvieron los valores más altos en el estrato arbóreo de PQ, en el arbustivo de Ppt y en el herbáceo de Pps. La prueba de Kruskal-Wallis (Tabla 2) indicó diferencias significativas entre todos los 
estratos de PQ y Pmn y solo entre los estratos arbustivo y herbáceo de Ppt. El índice de Simpson no presentó diferencias estadísticamente significativas entre el estrato arbóreo y herbáceo en ninguna asociación vegetal. Por el contrario, hubo diferencias significativas entre los estratos arbóreo-arbustivo y arbustivo-herbáceo en todas las asociaciones vegetales, con excepción de Ppt, para los árboles y los arbustos.
La mayor similitud, de acuerdo con el índice de Sorensen (Tabla 3), se obtuvo entre los estratos arbóreos de Pps y PQ (82\%) y la menor entre los de PQ y Pmn (59\%). En cuanto a los estratos de arbustos, los valores mayores de este índice se presentaron entre Pmn y Pps (80\%) y los menores entre PQ y Ppt (46\%); finalmente, en las herbáceas la mayor similitud en la composición de especies se registró entre Pmn y Ppt (66\%) y la menor entre Ppt y Pps (45\%).

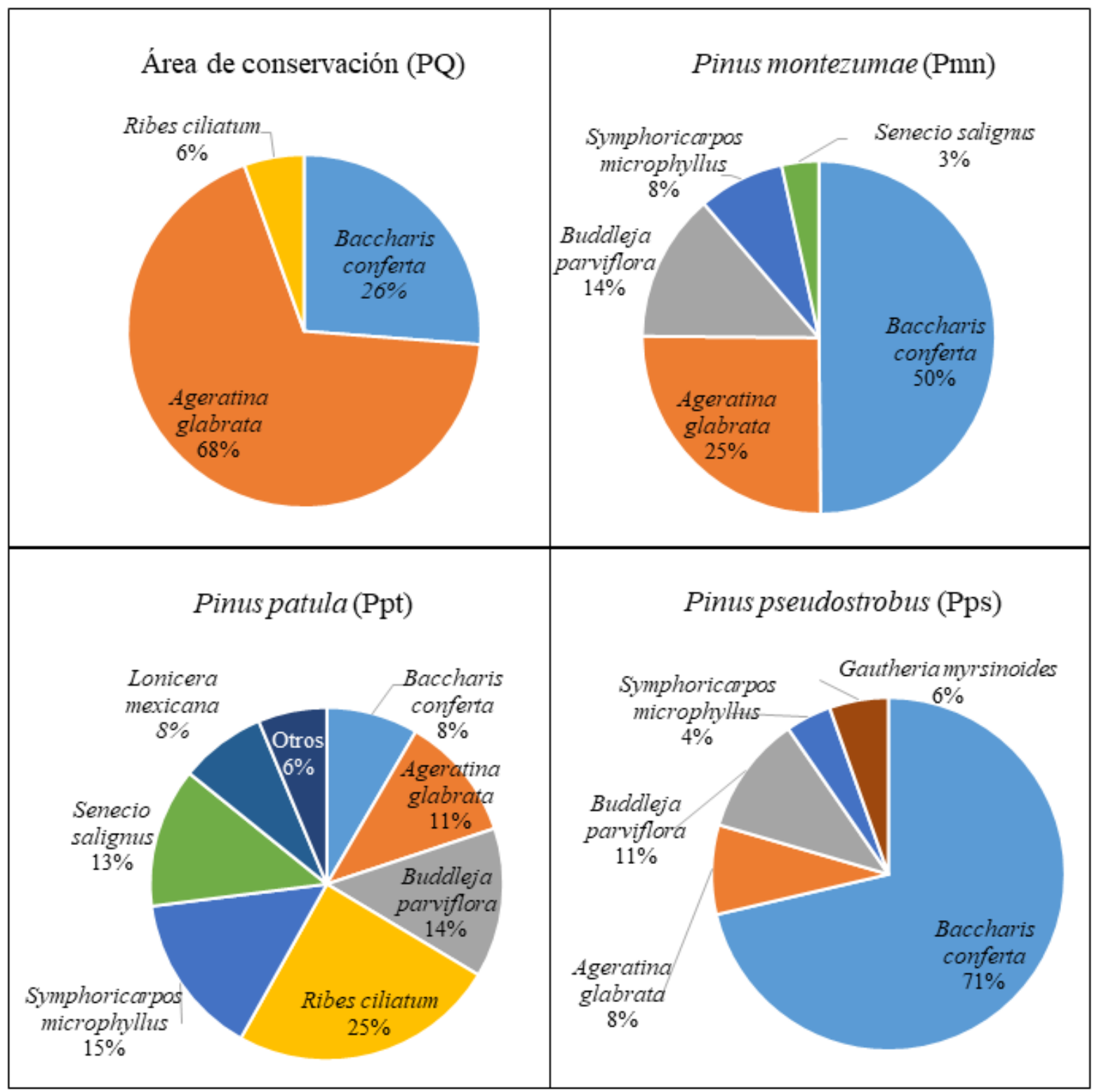

FIGURA 4. Porcentaje de importancia relativa por especie y asociación en el estrato arbustivo del ejido Nopalillo, Hidalgo, México. 


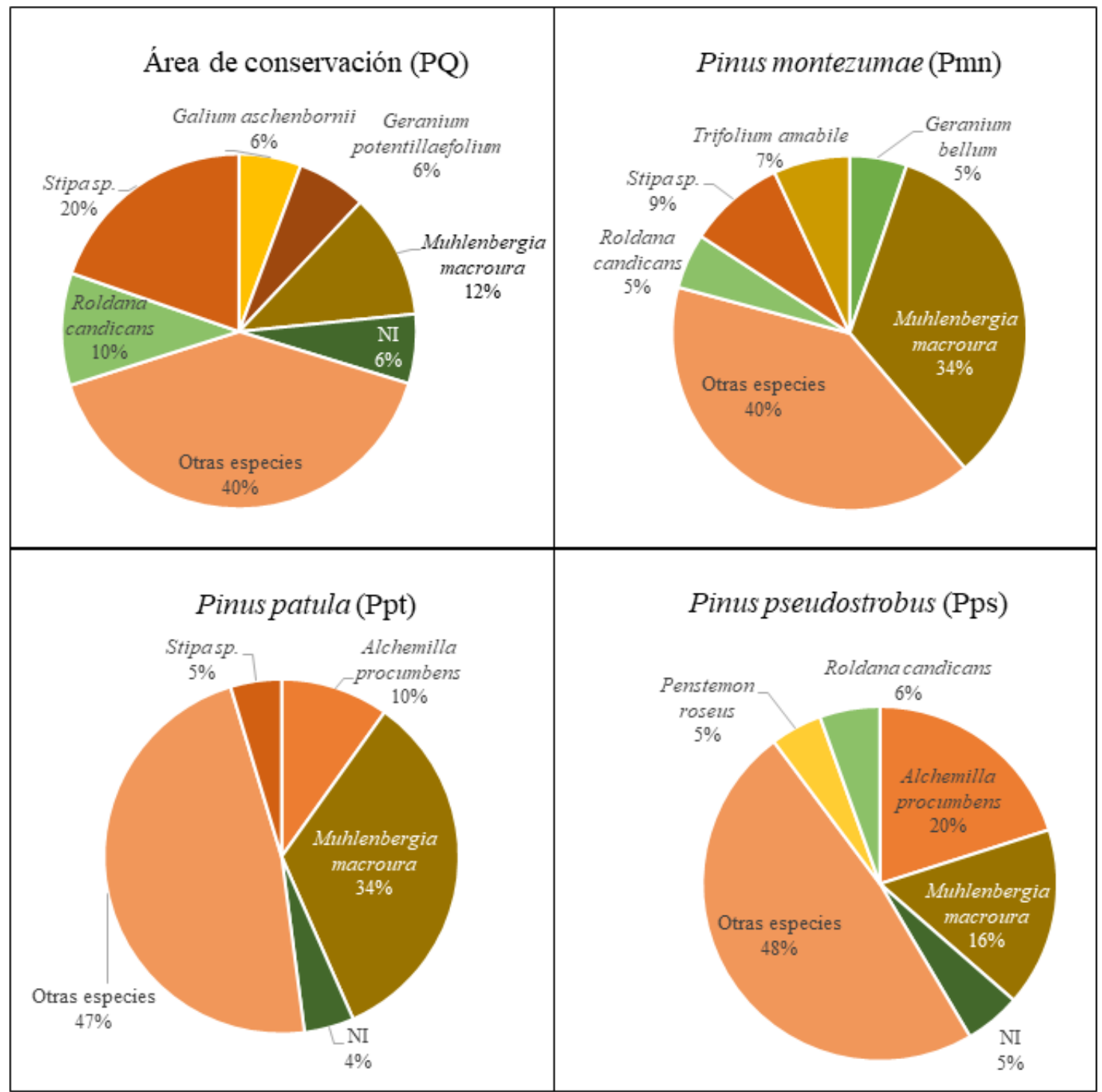

FIGURA 5. Porcentaje de importancia relativa por especie y asociación vegetal en el estrato herbáceo del ejido Nopalillo, Hidalgo, México.

Otras Especies: indice de valor de importancia es menor a 5\%, NI: Especie no identificada

TABLA 1. Diversidad alfa (índice de Margalef y Simpson) por estrato y asociación vegetal del bosque del ejido Nopalillo, Hidalgo, México.

\begin{tabular}{ccccccccc}
\hline \multirow{2}{*}{ Estrato } & \multicolumn{3}{c}{ Índice de Margalef } & \multicolumn{5}{c}{ Índice de Simpson } \\
& PQ & Pmn & Ppt & Pps & PQ & Pmn & Ppt & Pps \\
\hline Arbóreo & 1.24 & 0.63 & 0.59 & 0.75 & 0.65 & 0.35 & 0.26 & 0.47 \\
Arbustivo & 0.19 & 0.33 & 0.73 & 0.24 & 0.09 & 0.18 & 0.39 & 0.11 \\
Herbáceo & 0.78 & 0.86 & 0.63 & 0.87 & 0.59 & 0.55 & 0.43 & 0.62 \\
\hline
\end{tabular}

PQ: asociación Pinus-Quercus; Pmn: Pinus montezumae; Ppt: P. patula; Pps: P. pseudostrobus 
TABLA 2. Prueba de Kruskal-Wallis para el índice de Simpson y Margalef entre estratos dentro de cada asociación vegetal del ejido Nopalillo, Hidalgo, México.

\begin{tabular}{cccccccccc}
\hline \multirow{2}{*}{ Estratos } & \multicolumn{3}{c}{ Índice de Margalef } & \multicolumn{3}{c}{ Índice de Simpson } \\
& PQ & Pmn & Ppt & Pps & PQ & Pmn & Ppt & Pps \\
\hline Arbóreo-arbustivo & $0.002^{*}$ & $0.013^{*}$ & 0.43 & $0.010^{*}$ & $0.0002^{*}$ & $0.020^{*}$ & 0.7 & $0.0007^{*}$ \\
Arbóreo-herbáceo & $0.041^{*}$ & $0.013^{*}$ & 0.22 & 0.235 & 0.054 & 0.615 & 0.15 & 0.109 \\
Arbustivo-herbáceo & $0.001^{*}$ & $0.0006^{*}$ & $0.006^{*}$ & $0.006^{*}$ & $0.001^{*}$ & $0.007^{*}$ & $0.004^{*}$ & $0.004^{*}$ \\
\hline
\end{tabular}

PQ: Pinus-Quercus; Pmn: Pinus montezumae; Ppt: P. patula; Pps: P. pseudostrobus *Indica diferencia significativa $p<0.05$

TABLA 3. Índice de Sorensen por estrato y asociación vegetal en el ejido Nopalillo, Hidalgo, México.

\begin{tabular}{lccc}
\hline Asociaciones & $\begin{array}{c}\text { Arbóreo } \\
\%\end{array}$ & $\begin{array}{c}\text { Arbustivo } \\
\%\end{array}$ & $\begin{array}{c}\text { Herbáceo } \\
\%\end{array}$ \\
\hline PQ - Pmn & 0.59 & 0.50 & 0.50 \\
PQ - Ppt & 0.67 & 0.46 & 0.58 \\
PQ - Pps & 0.82 & 0.75 & 0.48 \\
Pmn - Ppt & 0.64 & 0.67 & 0.66 \\
Pmn - Pps & 0.76 & 0.80 & 0.55 \\
Ppt - Pps & 0.70 & 0.67 & 0.45 \\
\hline
\end{tabular}

PQ: asociación Pinus-Quercus; Pmn: Pinus montezumae; Ppt: P. patula; Pps: P. pseudostrobus

\section{Estructura horizontal y vertical}

Los individuos del estrato arbóreo se distribuyeron en mayor proporción en las primeras categorías diamétricas y su frecuencia disminuyó gradualmente conforme aumentó el diámetro (Fig. 6). PQ, Pmn y Ppt mostraron el mismo comportamiento, aunque con diferencias en el número de individuos por categorías. En PQ, 16\% de los registros correspondieron a la categoría de $35 \mathrm{~cm}$ o más de DN, mientras que para Pmn se trató de 9\% y en Ppt y Pps no superó $2 \%$ de los árboles medidos.

La distribución de individuos de acuerdo con su altura se concentró en las primeras tres categorías $(5 \mathrm{~m}-15 \mathrm{~m}$;
Fig. 7). En el caso de PQ, solo $20 \%$ de los registros superaron $15 \mathrm{~m}$ de altura; en Pmn fue 6\%, en Ppt 2\% y en Pps solo 1\%. Este patrón está relacionado directamente con la estructura horizontal, ya que diámetro y altura están correlacionados y, a su vez, lo están con la edad del arbolado (Smith, Larson, Kelty y Ashton et al., 1997). En Pmn, el arbolado se distribuyó hasta la categoría de $25 \mathrm{~m}$ y representó a los rodales más antiguos, en donde se realizaron las primeras cortas de regeneración. En Ppt y Pps, al ser rodales con las cortas más recientes, la máxima categoría registrada fue $20 \mathrm{~m}$. 


\section{Área de conservación (PQ)}

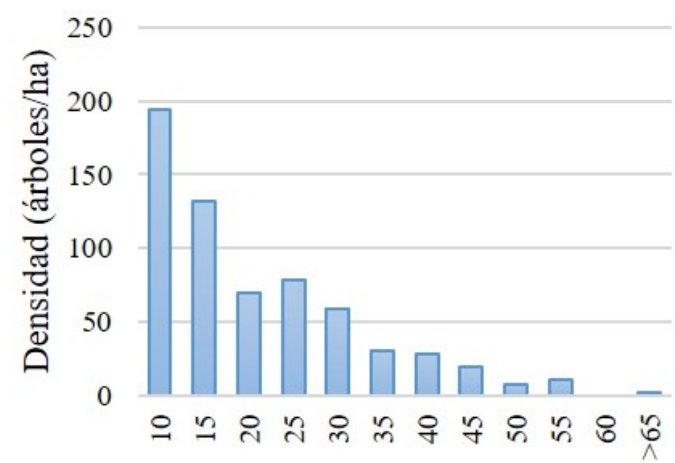

Categorías diamétricas $(\mathrm{cm})$

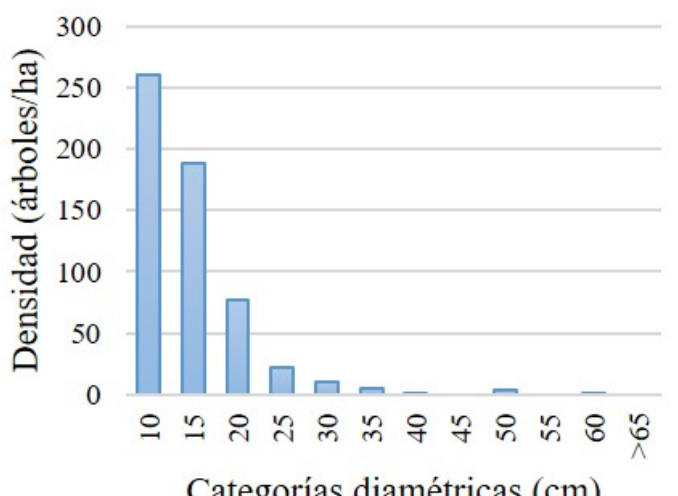

Categorías diamétricas $(\mathrm{cm})$

Pinus montezumae (Pmn)

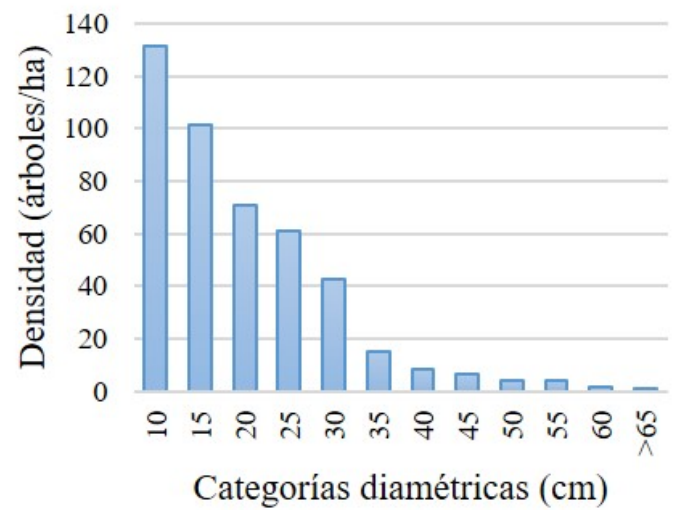

Pinus pseudostrobus (Pps)

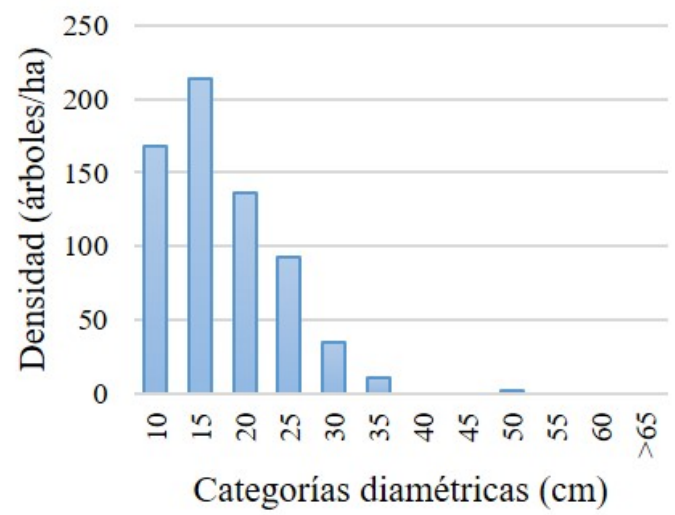

FiguRA 6. Distribución de categorías diamétricas (cm) para las cuatro asociaciones vegetales en el ejido Nopalillo, Hidalgo, México.

\section{Índice de valor de importancia}

La tabla 4 muestra que los valores del IVI para el estrato arbóreo difirieron en cada una de las asociaciones vegetales. En PQ, las especies de Pinus y Quercus registraron los mayores valores de importancia ecológica, acumulando $49 \%$ y $34 \%$, respectivamente, y el resto de las especies acumuló 17\% restante (Fig. 3). Esta asociación presentó una mayor mezcla de especies al compararse con las asociaciones bajo aprovechamiento maderable.

Considerando a todas las asociaciones, las especies más comunes fueron Alnus acuminata Kunth., Arbutus xalapensis Kunth., Pinus montezumae, P. patula Schiede ex Schltdl. \& Cham., P. pseudostrobus Lindl., Quercus crassifolia Bonpl. y Q. laurina Bonpl. Las especies exclusivas de Pmn fueron
Cupressus lusitanica Mill., Juniperus deppeana Steud., Quercus affinis Scheidw. y Pinus hartwegii Lindl.; en tanto que de Ppt fue Prunus serotina Ehrh. y de PQ, Salix paradoxa Kunth.

Los valores del IVI en el estrato arbustivo (Tabla 5) mostraron diferencias entre las asociaciones; por ejemplo, en Ppt se distribuyeron entre 10 especies, mientras que en PQ solo entre tres. Por otra parte, Baccharis conferta y Ageratina glabrata, además de ser las especies más comunes de las cuatro asociaciones, presentaron los valores IVI más altos (71\% y 50\%, respectivamente). Ppt presentó una distribución más equitativa de los valores del IVI, con Ribes ciliatum Humb. \& Bonpl. ex Roem. \& Schult. en primer lugar (24\%). 

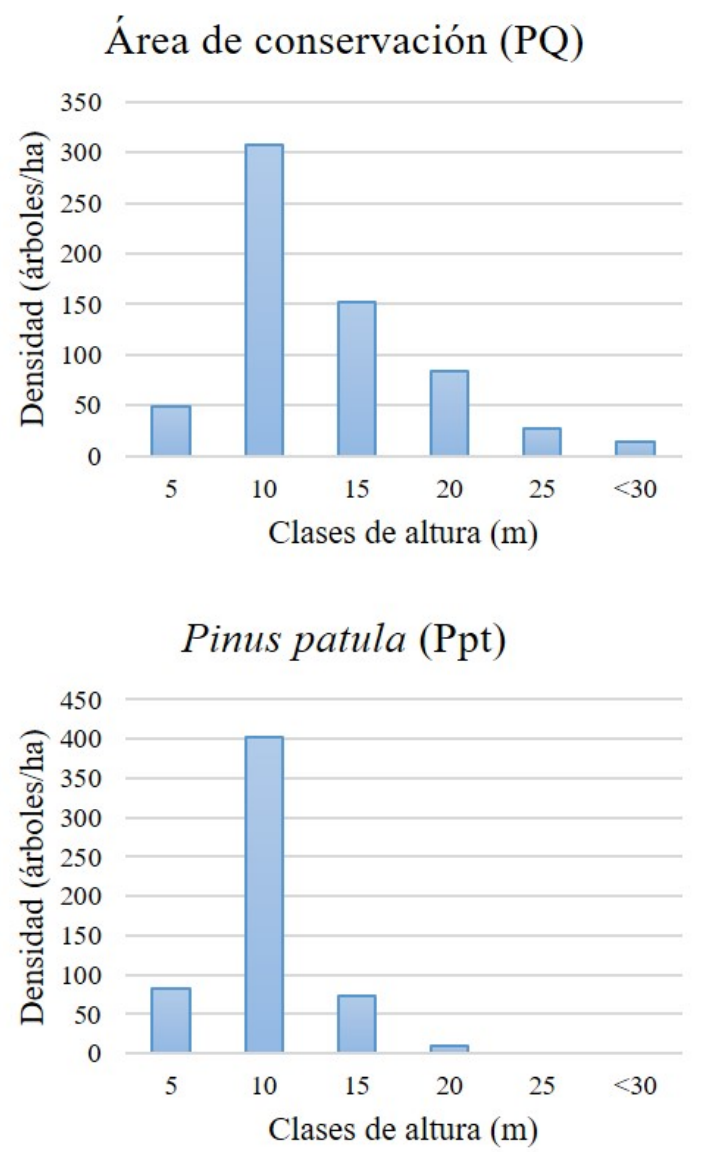

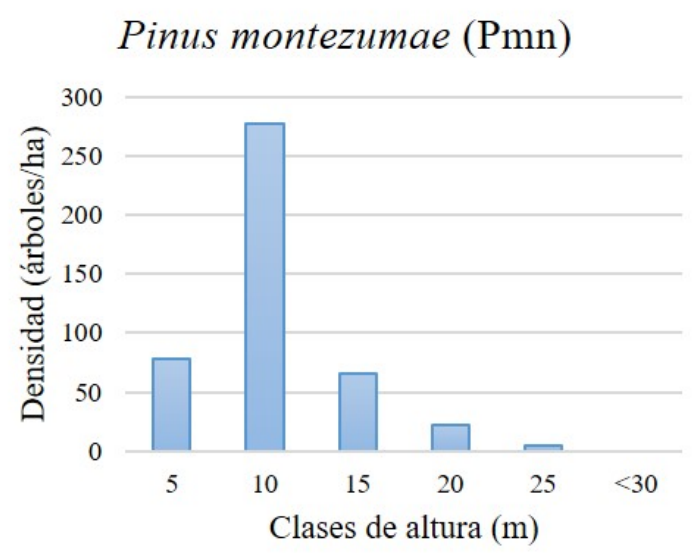

\section{Pinus pseudostrobus (Pps)}

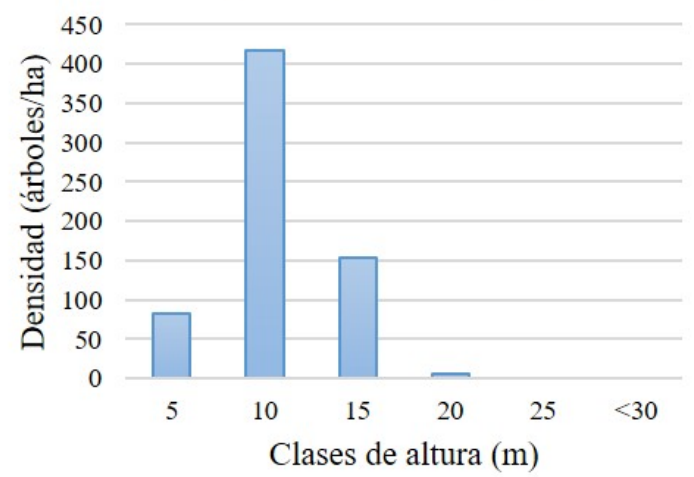

Figura 7. Distribución de clases de altura (m) para las cuatro asociaciones vegetales presentes en el ejido Nopalillo, Hidalgo, México.

TABLA 4. Índice de valor de importancia ecológica (IVI) del estrato arbóreo en el ejido Nopalillo, Hidalgo, México.

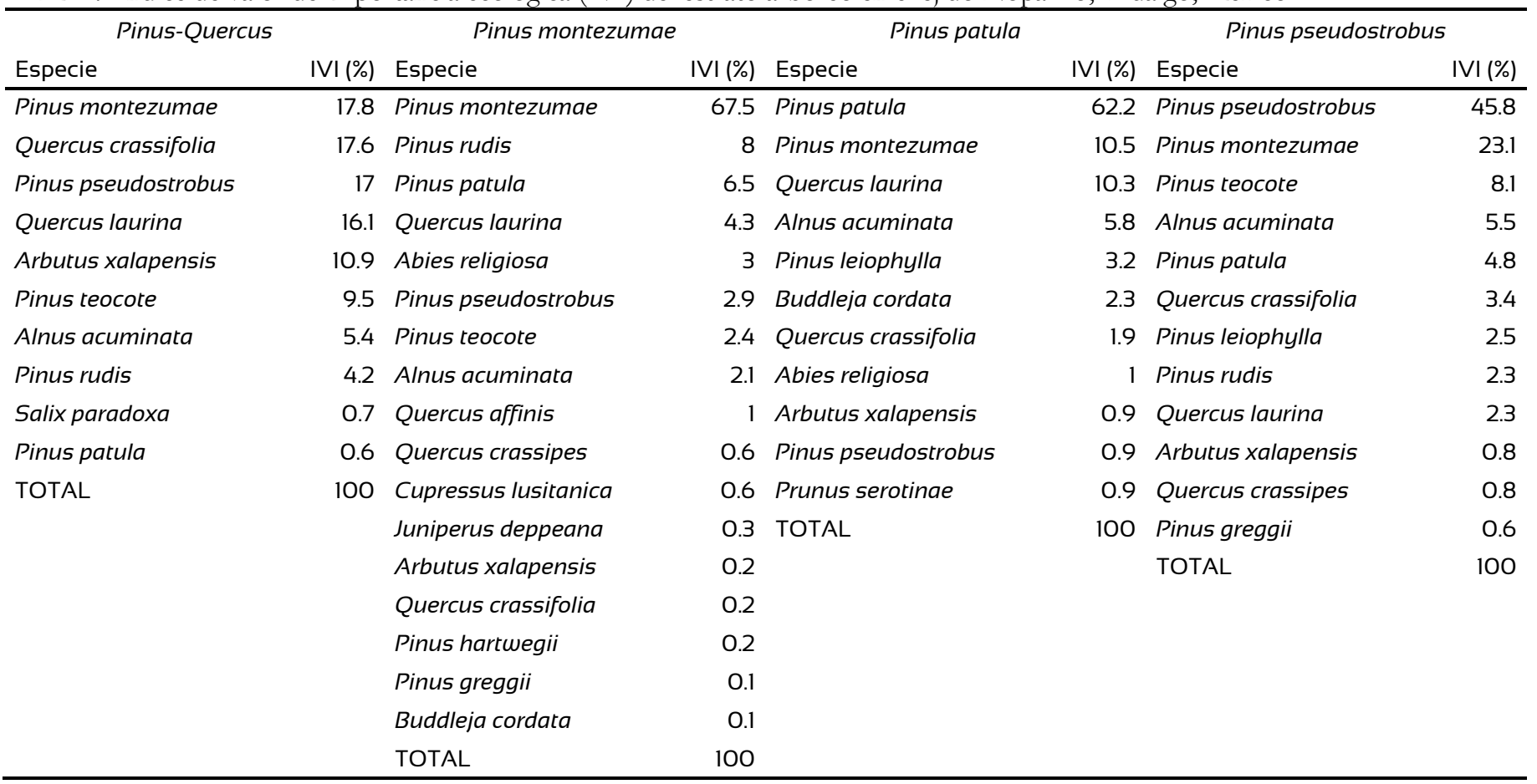


TABLA 5. Índice de valor de importancia relativa (IVI) del estrato arbustivo en el ejido Nopalillo, Hidalgo, México.

\begin{tabular}{|c|c|c|c|c|c|c|c|}
\hline \multirow{2}{*}{$\begin{array}{l}\text { Pinus-Quercus } \\
\text { Especie }\end{array}$} & \multicolumn{3}{|c|}{ Pinus montezumae } & \multirow{2}{*}{$\begin{array}{l}\text { Pinus patula } \\
\text { Especie }\end{array}$} & \multicolumn{3}{|c|}{ Pinus pseudostrobus } \\
\hline & $\begin{array}{l}\text { IVI } \\
\%\end{array}$ & Especie & $\begin{array}{l}\text { IVI } \\
\%\end{array}$ & & $\begin{array}{l}\text { IVI } \\
\%\end{array}$ & Especie & $\begin{array}{l}\text { IVI } \\
\%\end{array}$ \\
\hline $\begin{array}{l}\text { Ageratina } \\
\text { glabrata }\end{array}$ & 68.3 & Baccharis conferta & 49.9 & Ribes ciliatum & 24.5 & Baccharis conferta & 71.4 \\
\hline $\begin{array}{l}\text { Baccharis } \\
\text { conferta }\end{array}$ & 26.2 & Ageratina glabrata & 25.3 & $\begin{array}{l}\text { Symphoricarpos } \\
\text { microphyllus }\end{array}$ & 14.9 & Buddleja parviflora & 10.9 \\
\hline Ribes ciliatum & 5.5 & Buddleja parviflora & 13.6 & Buddleja parviflora & 13.7 & Ageratina glabrata & 8.2 \\
\hline \multirow[t]{8}{*}{ TOTAL } & 100 & $\begin{array}{l}\text { Symphoricarpos } \\
\text { microphyllus }\end{array}$ & 7.9 & Senecio salignus & 12.8 & Gaultheria myrsinoides & 5.4 \\
\hline & & Senecio salignus & 3.4 & Ageratina glabrata & 11.5 & $\begin{array}{l}\text { Symphoricarpos } \\
\text { microphyllus }\end{array}$ & 4.2 \\
\hline & & TOTAL & 100 & Baccharis conferta & 8.4 & TOTAL & 100 \\
\hline & & & & Lonicera mexicana & 7.9 & & \\
\hline & & & & Litsea glaucescens & 3.7 & & \\
\hline & & & & Arctostaphylos pungens & 1.3 & & \\
\hline & & & & Gaultheria myrsinoides & 1.3 & & \\
\hline & & & & TOTAL & 100 & & \\
\hline
\end{tabular}

Finalmente, en el estrato herbáceo (Tabla 6) el IVI mostró que en las asociaciones bajo aprovechamiento maderable dos especies fueron las dominantes (Mulbembergia macroura y Lachemilla procumbens), con importancias ecológicas acumuladas entre $36 \%$ y $43 \%$. En este estrato también se encontró la mayor cantidad de especies, aunque en su mayoría con valores de IVI menores a 5\%.

\section{DISCUSIÓN}

\section{Composición, riqueza y diversidad}

En el presente estudio se encontraron menos especies (20) que las registradas por Castellanos-Bolaños et al. (2008) para un bosque de Pinus patula en Oaxaca, (26). Sin embargo, la cifra supera el intervalo de 11 a 18 especies obtenidas en el estrato arbóreo de bosques templados de pino-encino y encino-pino bajo aprovechamiento maderable (Domínguez Gómez et al. 2018; Zacarias-Eslava, Cornejo-Tenorio, Cortés-Flores, González-Castañeda e Ibarra-Manríquez, 2011; Delgado Zamora et al., 2017; Graciano-Ávila et al., 2017; López-Hernández et al., 2017; González-Tagle, Scwendenmann, Jiménez-Pérez y Schulz, 2008; MonarrezGonzalez et al., 2020).

En el estrato arbustivo, Vázquez-Cortes et al. (2018) registraron 12 especies para un bosque de Oaxaca a partir de sitios donde se aplicó matarrasa, en los bordes de estas áreas y en sitios sin corta. Luna-Bautista et al. (2015) encontraron que el estrato arbustivo es uno de los que más contribuyen a la riqueza y diversidad de especies del bosque de Pinus oaxacana Mirov. en Oaxaca, y que incluso pueden presentar índices de diversidad mayores al de los árboles. 
Madera y Bosques vol. 27, núm. 1, e2712127 Primavera 2021

TABLA 6. Índice de valor de importancia relativa (IVI) del estrato herbáceo en el ejido Nopalillo, Hidalgo, México.

\begin{tabular}{|c|c|c|c|}
\hline \multirow{2}{*}{$\begin{array}{l}\text { Pinus-Quercus } \\
\text { Especie }\end{array}$} & \multirow[b]{2}{*}{ IVI (\%) } & \multicolumn{2}{|l|}{ Pinus montezumae } \\
\hline & & Especie & IVI (\%) \\
\hline Stipa spp. & 19.7 & Muhlenbergia macroura & 33.5 \\
\hline Muhlenbergia macroura & 11.4 & Stipa spp & 8.8 \\
\hline Roldana candicans & 10.3 & Trifolium amabile & 7 \\
\hline $\mathrm{NI}-8$ & 6.3 & Geranium bellum & 5.2 \\
\hline Geranium potentillifolium & 6.3 & Roldana candicans & 5 \\
\hline Galium aschenbornii & 5.7 & Geranium potentillifolium & 3.9 \\
\hline $\mathrm{NI}-27$ & 3.9 & Penstemon roseus & 3.6 \\
\hline Physalis coztomatl & 3.6 & Eryngium monocephalum & 3.2 \\
\hline $\mathrm{NI}-11$ & 3.5 & Lupinus montanus & 2.7 \\
\hline Geranium bellum & 3.3 & Alchemilla procumbens & 2.6 \\
\hline Didymaea alsinoides & 3.2 & Lithospermum distichum & 2.5 \\
\hline Verbena bippinatifida & 3.2 & $\mathrm{NI}-2$ & 2.1 \\
\hline Chimaphylla umbellata & 2.7 & $\mathrm{NI}-7$ & 2.1 \\
\hline $\mathrm{NI}-31$ & 2.1 & Penstemon hartwegii & 2 \\
\hline Eryngium monocephalum & 1.8 & Verbena bippinatifida & 2 \\
\hline${ }^{*}$ Otras especies & 13.2 & ${ }^{*}$ Otras especies & 13.8 \\
\hline Total & 100 & Total & 100 \\
\hline Pinus pseudostrobus & & Pinus patula & \\
\hline Especie & IVI (\%) & Especie & IVI (\%) \\
\hline Lachemilla procumbens & 20.1 & Muhlenbergia macroura & 33.5 \\
\hline Muhlenbergia macroura & 16.3 & Lachemilla procumbens & 9.9 \\
\hline Roldana candicans & 5.5 & Stipa spp & 4.6 \\
\hline $\mathrm{NI}-8$ & 5.1 & $\mathrm{NI}-8$ & 4.5 \\
\hline Penstemon roseus & 4.7 & Roldana candicans & 3.8 \\
\hline Geranium potentillifolium & 4.1 & Galium aschenbornii & 3.7 \\
\hline Stipa spp & 4.1 & Oxalis divergens & 3.5 \\
\hline Geranium bellum & 3.2 & $\mathrm{NI}-18$ & 2.9 \\
\hline Oxalis divergens & 3.2 & Fragaria mexicana & 2.8 \\
\hline Verbena bippinatifida & 2.9 & Ribes ciliatum & 2.8 \\
\hline Galium aschenbornii & 2.7 & Geranium bellum & 2.5 \\
\hline Fragaria mexicana & 2.5 & Penstemon roseus & 2.3 \\
\hline Lonicera mexicana & 2.4 & Lonicera mexicana & 2.2 \\
\hline Eryngium monocephalum & 2.2 & Verbena bippinatifida & 2 \\
\hline $\mathrm{NI}-4$ & 2.1 & Geranium potentillifolium & 1.9 \\
\hline${ }^{*}$ Otras especies & 19 & *Otras especies & 16.9 \\
\hline Total & 100 & Total & 100 \\
\hline
\end{tabular}


Con respecto a la diversidad alfa, en el presente trabajo se encontró un valor máximo del índice de Margalef de 1.24 para el área de conservación (PQ); por consiguiente, presentó mayor riqueza que las áreas bajo aprovechamiento (0.63 - 0.75) o que las estudiadas por Návar-Chaidez y González-Elizondo (2009), Hernández-Salas et al. (2013) para áreas bajo aprovechamiento maderable en el noroeste de México, con valores de 1.0 y $0.81-0.90$, respectivamente. Ahora bien, Graciano-Ávila et al. (2017) y López-Hernández et al. (2017) en Puebla, México, encontraron mayor diversidad con valores de 1.58 y 1.34 , respectivamente.

El índice de Simpson máximo de este trabajo se encontró en el estrato herbáceo del área bajo aprovechamiento maderable (0.62), mientras que el valor más alto del arbolado fue para el área de conservación (0.65). Zacarías-Eslava et al. (2011) obtuvieron valores de 0.18 a 0.45 para el estrato arbóreo, similares a los encontrados en este estudio para el área bajo aprovechamiento maderable.

Las investigaciones en bosques manejados en México, generalmente, no incluyen información sobre el sotobosque, no obstante que Luna-Bautista et al. (2015) lo señalan como el de mayor riqueza y diversidad, independientemente del tratamiento silvícola y tiempo de aplicación analizados.

\section{Estructura horizontal y vertical}

La estructura de J invertida en la distribución diamétrica se debe, en parte, a que los pinos se establecen al abrirse claros como consecuencia de las cortas de regeneración y muestran la reposición del piso forestal después de la corta. Este tipo de distribución ha sido observada en estudios realizados en bosques templados de pino y pino-encino bajo aprovechamiento de Puebla, Oaxaca y Durango (SolísMoreno et al., 2006; Castellanos-Bolaños et al., 2008; NávarChaidez y González-Elizondo, 2009; Luna-Bautista et al., 2015; Delgado-Zamora et al., 2017; Graciano-Ávila et al., 2017; López-Hernández et al., 2017; Medrano Meraz et al., 2017; Vásquez-Cortez et al., 2018).

En el caso de PQ, la ausencia de cortas de regeneración desde hace 50 años permite que haya una mayor cantidad de arbolado de grandes dimensiones (Asociación de Silvicultures de la Región Forestal Pachuca-Tualancingo, A.C., 2015), al compararse con las otras asociaciones. Esta es una característica propia de los bosques naturales o que no han tenido disturbios frecuentes (Smith et al., 1997). La estructuras horizontal y vertical de PQ, la ausencia de registros de arbolado con edad o dimensiones considerables y el historial de disturbios de la región indican que se trata de un bosque secundario (Chokkalingam y de Jong, 2001).

Las diferencias en la distribución de diámetros y alturas dentro de cada asociación vegetal pueden deberse al tiempo transcurrido desde la aplicación de las cortas de regeneración. Las intervenciones silvícolas más recientes se realizaron en Ppt y Pps y las más antiguas en Pmn (Asociación de Silvicultures de la Región Forestal PachucaTualancingo, A.C., 2015).

\section{Índice de valor de importancia}

En las asociaciones bajo aprovechamiento maderable se encontró mayor dominancia de Pinus, lo que muestra que el bosque del área de conservación es más heterogéneo en su composición de especies. Esta misma situación ha sido observada en otras áreas bajo manejo en Durango (Graciano-Ávila et al., 2017), Chihuahua (López-Hernández et al., 2017), Puebla (Hernández-Salas et al., 2012) y Oaxaca (Castellanos-Bolaños et al., 2008), donde las prácticas silvícolas han favorecido al género Pinus. Este tipo de patrón es esperado de acuerdo con los objetivos y método de manejo aplicado en el área, ya que a través de cortas de regeneración de árboles padre se busca obtener bosques regulares monoespecíficos y coetáneos. Los aclareos no comerciales y comerciales aplicados posteriormente contribuyen a lograr estos objetivos, reflejándose en un IVI de más de $70 \%$ para Pinus en las tres asociaciones con manejo y de, al menos, $45 \%$ para la especie que define la asociación vegetal.

Como se observó en este estudio, los valores de IVI y la composición taxonómica de las asociaciones reflejan el efecto de las prácticas silvícolas. Sin embargo, sería recomendable considerar también las características del 
sitio, tales como la altitud, pendiente y exposición, pues son determinantes de la dominancia estructural (CastellanosBolaños et al., 2008; Graciano-Ávila et al., 2017).

El sotobosque (estratos arbustivo y herbáceo) es un componente de los bosques templados que concentra una alta diversidad (Luna-Bautista et al., 2015; Mejía-Canales, FrancoMaas, Endara, Ávila, 2018), además de ser un elemento clave para el ciclo de nutrientes a partir de la incorporación de materia orgánica al suelo, como hábitat y fuente de alimento para la fauna o protección física al suelo (Guillian, 2007).

En el estrato arbustivo se encontraron algunas especies pioneras que aparecen y se desarrollan posteriormente a un disturbio, por ejemplo, Baccharis conferta, Senecio salignus DC. y Arctostaphylos pungens Kunth (Márquez et al., 1999). Las diferencias en la composición del sotobosque están relacionadas con las características del dosel, ya que este impacta en la cantidad de luz disponible y, por lo tanto, en las especies que pueden proliferar (Ross, Flanagan y La Roi, 1986). Por consiguiente, la incidencia de disturbios, como las cortas de regeneración y los aclareos, tiene efectos sobre el sotobosque; del mismo modo las características del sitio, como la pendiente y la exposición, se relacionan con su abundancia (Stephen y Chen, 2006). Estos resultados son consistentes con la composición, la riqueza y la diversidad de las especies, así como con el valor de importancia de los estratos en cada asociación.

\section{CONCLUSIONES}

Debido a las diferencias encontradas en composición, diversidad y estructura entre las condiciones bajo aprovechamiento maderable no es posible considerarlas menos complejas y diversas que el área de conservación. Por lo anterior, las prácticas silvícolas realizadas en el bosque analizado contribuyen al logro de un manejo forestal sustentable.

Las especies arbóreas con mayor contribución estructural en el estrato arbóreo fueron Pinus montezumae, $P$. patula, P. pseudostrobus, Quercus laurina y Q. crassifolia; en el estrato arbustivo Baccharis conferta, Ageratina glabatra y Ribes ciliatun $\mathrm{y}$, dentro de las herbáceas, el mayor componente estructural estuvo representado por Mublenbergia macroura, Lachemilla procumbens y Stipa sp.

La mayor diversidad de especies del estrato arbustivo y herbáceo se encontró en las condiciones bajo aprovechamiento maderable y, para el estrato arbóreo, en el área sin manejo. Los resultados de este trabajo ofrecen un referente para contrastar los cambios que experimenta el bosque a través del tiempo a causa de disturbios naturales o de las prácticas silvícolas y podrán representar la base para el desarrollo de estrategias de manejo, conservación y uso del bosque.

\section{RECONOCIMIENTOS}

Al Consejo Nacional de Ciencia y Tecnología por la beca otorgada a la primera autora para realizar estudios de maestría. A la asamblea y los representantes del ejido El Nopalillo, Hidalgo que permitieron la realización de esta investigación en su bosque y a las familias Montiel Castelán y Vera Castelán por toda la ayuda en el trabajo de campo.

ANEXO 1. Especies arbóreas, arbustivas y herbáceas registradas en el bosque del ejido Nopalillo, Hidalgo, México.

\begin{tabular}{llll}
\hline Forma de vida & Familia & Especie* $^{*}$ & Autoridad \\
\hline Árbol & Asparagaceae & Furcraea parmentieri & (Roexl) García-Mend. \\
Árbol & Betulaceae & Alnus acuminata & Kunth \\
Árbol & Cupressaceae & Cupressus lusitanica & Mill. \\
Árbol & Cupressaceae & Juniperus deppeana & Steud. \\
Árbol & Ericaceae & Arbutus xalapensis & Kunth \\
Árbol & Fagaceae & Quercus affinis & Scheidw. \\
Árbol & Fagaceae & Quercus crassifolia & Bonpl. \\
Árbol & Fagaceae & Quercus crassipes & Bonpl. \\
Árbol & Fagaceae & Quercus laurina & Bonpl.
\end{tabular}


ANEXO 1. Especies arbóreas, arbustivas y herbáceas registradas en el bosque del ejido Nopalillo, Hidalgo, México.

\begin{tabular}{|c|c|c|c|}
\hline Forma de vida & Familia & Especie* $^{*}$ & Autoridad \\
\hline Árbol & Pinaceae & Abies religiosa & (Kunth) Schltdl. \& Cham. \\
\hline Árbol & Pinaceae & Pinus greggii & Engelm. ex Parl. \\
\hline Árbol & Pinaceae & Pinus hartwegii & Lindl. \\
\hline Árbol & Pinaceae & Pinus leiophylla & Schiede ex Schltdl. \& Cham. \\
\hline Árbol & Pinaceae & Pinus montezumae & Lamb. \\
\hline Árbol & Pinaceae & Pinus patula & Schiede ex Schltdl. \& Cham. \\
\hline Árbol & Pinaceae & Pinus pseudostrobus & Lindl. \\
\hline Árbol & Pinaceae & Pinus teocote & Schiede ex Schltdl. \& Cham \\
\hline Árbol & Rosaceae & Prunus serotina & Ehrh. \\
\hline Árbol & Salicaceae & Salix paradoxa & Kunth \\
\hline Árbol & Scrophulariaceae & Buddleja cordata & Kunth \\
\hline Arbusto & Asteraceae & Ageratina glabrata & Kunth \\
\hline Arbusto & Asteraceae & Baccharis conferta & Kunth \\
\hline Arbusto & Asteraceae & Senecio salignus & $\mathrm{DC}$ \\
\hline Arbusto & Caprifoliaceae & Lonicera mexicana & (Kunth) Rehder \\
\hline Arbusto & Caprifoliaceae & Symphoricarpos microphyllus & Kunth \\
\hline Arbusto & Ericaceae & Arctostaphylos pungens & Kunth \\
\hline Arbusto & Ericaceae & Gaultheria myrsinoides & Kunth \\
\hline Arbusto & Grossulariaceae & Ribes ciliatum & Humb. \& Bonpl. ex Roem. \& Schult. \\
\hline Arbusto & Lauraceae & Litsea glaucescens & Kunth \\
\hline Arbusto & Scrophulariaceae & Buddleja parviflora & Kunth \\
\hline Hierba & Apiaceae & Eryngium monocephalum & Cav. \\
\hline Hierba & Asteraceae & Dahlia scapigera & (A.Dietr.) Knowles \& Westc. \\
\hline Hierba & Asteraceae & Cirsium ehrenbergii & Sch.Bip. \\
\hline Hierba & Asteraceae & Roldana candicans & (Née) Villaseñor, S. Valencia \& Coombes \\
\hline Hierba & Asteraceae & Seneciol sp & L. \\
\hline Hierba & Asteraceae & Senecio2 sp & $\mathrm{L}$. \\
\hline Hierba & Asteraceae & Stevial sp & Cav. \\
\hline Hierba & Asteraceae & Stevia2 sp & Cav. \\
\hline Hierba & Boraginaceae & Lithospermum distichum & Ortega \\
\hline Hierba & Caprifoliaceae & Lonicera mexicana & (Kunth) Rehder \\
\hline Hierba & Caryophyllaceae & Arenaria lycopodioides & Willd. ex Schltdl. \\
\hline Hierba & Ericaceae & Chimaphila umbellata & (L.) Nutt. \\
\hline Hierba & Geraniaceae & Geranium bellum & Rose \\
\hline Hierba & Geraniaceae & Geranium seemannii & Peyr. \\
\hline Hierba & Geraniaceae & Geranium potentillifolium & DC. \\
\hline Hierba & Grossulariaceae & Ribes ciliatum & Humb. \& Bonpl. ex Roem. \& Schult. \\
\hline Hierba & Lamiaceae & Salvia laevis & Benth. \\
\hline Hierba & Leguminosae & Lupinus montanus & Kunth \\
\hline Hierba & Leguminosae & Trifolium amabile & Kunth \\
\hline Hierba & Lentibulariaceae & Pinguicola moranensis & Kunth \\
\hline Hierba & Orobanchaceae & Castilleja scorzonerifolia & Kunth \\
\hline Hierba & Rosaceae & Fragaria vesca & $\mathrm{L}$. \\
\hline Hierba & Rosaceae & Lachemilla procumbens & Rose \\
\hline Hierba & Rubiaceae & Didymaea alsinoides & (Cham. \& Schltdl.) Standl. \\
\hline Hierba & Rubiaceae & Galium aschenbornii & Schauer \\
\hline Hierba & Onagraceae & Fuchsia thymifolia & Kunth \\
\hline Hierba & Orchidaceae & Goodyera striata & Rchb.f. \\
\hline Hierba & Orobanchaceae & Lamourouxia multifida & Kunth \\
\hline
\end{tabular}


ANEXO 1. Especies arbóreas, arbustivas y herbáceas registradas en el bosque del ejido Nopalillo, Hidalgo, México.

\begin{tabular}{llll}
\hline Forma de vida & Familia & Especie* $^{*}$ & Autoridad \\
\hline Hierba & Oxalidaceae & Oxalis divergens & Benth. ex Lindl. \\
Hierba & Plantaginaceae & Penstemon campalunatus & (Cav.) Willd. \\
Hierba & Plantaginaceae & Penstemon hartwegii & Benth. \\
Hierba & Plantaginaceae & Penstemon roseus & (Cerv. ex Sweet) G. Don \\
Hierba & Poaceae & Muhlenbergia macroura & (Humb., Bonpl. \& Kunth) Hitchc. \\
Hierba & Poaceae & Stipa sp & L. \\
Hierba & Solanaceae & Physalis coztomatl & Dunal \\
Hierba & Solanaceae & Solanum nigrescens & M. Martens \& Galeotti \\
Hierba & Verbenaceae & Verbena bippinatifida & Nutt. \\
Hierba & Verbenaceae & Verbena recta & Kunth \\
\hline
\end{tabular}

* No se incluyen 8 taxones identificados solo a nivel de Familia (Asteraceae) y 12 especies más sin identificar

\section{REFERENCIAS}

Asociación de Silvicultores de la Región Forestal Pachuca-Tualancingo, A. C. (2015). Programa de Manejo Forestal, nivel avanzado, para el ejido Nopalillo, Epazoyucan, Hidalgo. Reporte Técnico. 169 p.

Calderón de Rzedowski, G. de \& Rzedowski, J. (2005). Flora fanerogámica del Valle de México (2a. ed.), versión digital., Pátzcuaro, México: Instituto de Ecología, A.C. y Comisión Nacional para el Conocimiento y Uso de la Biodiversidad, Pátzcuaro (Michoacán). Recuperado de https://www.biodiversidad.gob.mx/publicaciones/librosDig/p df/Flora_del_Valle_de_Mx1.pdf

Castellanos-Bolaños, J. F., Treviño-Garza, E. J., Aguirre-Calderón, Ó. A., Jiménez-Pérez, J., Musalem-Santiago, M., \& López-Aguillón, R. (2008). Estructura de bosques de pino pátula bajo manejo en Ixtlán de Juárez, Oaxaca, México. Madera y Bosques, 14(2), 51-63. doi: $10.21829 /$ myb.2008.1421212

Corral Rivas, J. J., Aguirre Calderón, O. A., Jiménez Pérez, J., \& Corral Rivas, S. (2005). Un análisis del efecto del aprovechamiento forestal sobre la diversidad estructural en el Bosque Mesófilo de Montaña" El Cielo", Tamaulipas, México. Investigación Agraria. Sistemas y Recursos Forestales, 14(2), 217-228. Recuperado de http://www.inia.es/gcontrec/pub/217-228-(30_0S)-

Un_analisis_1162281437750.pdf

Chokkalingam,U. and de Jong, W. 2001. Secondary forest: a working definition and typology International Forestry Review, 3(1), 19-26. Recuperado de www.jstor.org/stable/42609342?seq=1

Comisión Nacional Forestal [Conafor] (2014). Inventario estatal forestal. Hidalgo. Zapopan., Jalisco. 172 p.

Comisión Nacional Forestal [Conafor] (2017). Inventario nacional forestal y de suelos. Informe de resultados 2009-2014. Zapopan, Jalisco. 200 p.
Delgado Zamora, D. A., Heynes Silerio, S. A., Mares Quiñones, M. D., Piedra Leandro, N. L., Retana Rentería, F. I., Rodríguez Corral, K., .., \& Ruacho-González, L. (2017). Diversidad y estructura arbórea de dos rodales en Pueblo Nuevo, Durango. Revista Mexicana de Ciencias Forestales, 7(33), 094-107. doi: $10.29298 /$ rmcf.v7i33.92

Del Río M., F. Montes, I. Cañellas, \& G. Montero. (2003). Revisión: índices de diversidad estructural en masas forestales. Investigación Agraria. Sistemas y recursos forestales, 12(1), 159-176.

Domínguez Gómez, G. T., Hernández González, B. N., González Rodriguez, H., Cantú Silva, I., Alanís Rodriguez, E., \& Alvarado, M. del S. (2018). Estructura y composición de la vegetación en cuatro sitios de la Sierra Madre Occidental. Revista Mexicana de Ciencias Forestales, 9(50), 9-34. doi: 10.29298/rmcf.v9i50.227

Franklin, J. F., Spies, T. A., Pelt, R. Van, Carey, A. B., Thornburgh, D. A., Berg, D. R., ..., \& Chen, J. (2002). Disturbances and structural development of natural forest ecosystems with silvicultural implications, using Douglas-fir forests as an example. Forest Ecology and Management, 155, 399-423. doi: 10.1016/S03781127(01)00575-8

Gadow, K. von \& Hui, G. (1999). Modelling stand development. En K. von Gadow \& G. Hui (eds.), Modelling forest development. Forestry Science, Vol. 57. Switzerland: Springer. doi: 10.1007/978-94-0114816-0_3

Gilliam, F. S. (2007). The ecological significance of the herbaceous layer in temperate forest ecosystems. BioScience, 57(10), 845-858. doi: 10.1641/B571007

González Elizondo, M. S. y González Elizondo, M. (2014). Familia Ericaceae. Flora del Bajio y de Regiones Adyacentes, 183.

González-Tagle, M. A., Schwendenmann, L., Jiménez-Pérez, J., \& Schulz, R. (2008). Forest structure and woody plant species composition along a fire chronosequence in mixed pine-oak 
forest in the Sierra Madre Oriental, northeast Mexico. Forest Ecology and Management, 256, 161-167. doi:10.1016/j.foreco.2008.04.021

Graciano-Ávila, G., Alanís-Rodríguez, E., Aguirre-Calderón, O. A., González-Tagle, M. A., Treviño-Garza, E. J., \& Mora-Olivo, A. (2017). Caracterización estructural del arbolado en un ejido forestal del noroeste de México. Madera y Bosques, 23(3), 137-146. doi: $10.21829 /$ myb.2017.2331480

Hernández-Salas, J., Aguirre-Calderón, O. A., Alanís-Rodríguez, E., Jiménez-Pérez, J., Treviño-Garza, E. J., González-Tagle, M. A., .., \& Domínguez-Pereda, L. A. (2013). Efecto del manejo forestal en la diversidad y composición arbórea de un bosque templado del noroeste de México. Revista Chapingo, Serie Ciencias Forestales y del Ambiente, 19(2), 189-199. doi: 10.5154/r.rchscfa.2012.08.052

Jiménez, B. I., Damoa, A., Ochoa-Gaona, S., \& Clak, T. R.(2014). Impact of silvicultural methods on vascular epiphytes (ferns, bromeliad and orchids) in a temperate forest in Oaxaca, Mexico. Forest Ecology and Management, 329, 10-20. doi: 10.1016/j.ecolind.2016.02.004

Kimmins, J. P. (1997). Forest Ecology. A Foundation for Sustainable Management. Upper Saddle River, New Jersey: Prentice Hall. 611 p.

Koleff, P., Gaston, K. J., \& Lennon, J. J. (2003). Measuring beta diversity for presence-absence data. Journal of Animal Ecology, 72(3), $367-$ 382. doi: 10.1046/j.1365-2656.2003.00710.x

López-Hernández, J. A., Aguirre-Calderón, Ó. A., Alanís-Rodríguez, E., Monárrez-González, J. C., González-Tagle, M. A., \& JiménezPérez, J. (2017). Composición and diversidad de especies forestales en bosques templados de Puebla, México. Madera y Bosques, 23(1), 39-51. doi: 10.21829/myb.2017.2311518

Luna-Bautista, L., Hernández-de la Rosa, P., Velázquez-Martínez, A., Gómez-Guerrero, A., \& Acosta-Mireles, M. (2015). El sotobosque en la composición y diversidad de áreas bajo manejo forestal en Santa Catarina Ixtepeji, Oaxaca. Revista Chapingo, Serie Ciencias Forestales y del Ambiente, 21(1), 109-121. doi: 10.5154/r.rchscfa.2014.08.037

Magurran, A. E. (2004). Measuring Biological Diversity. (9a ed.). United Kingdom: Blackwell Publishing Company.

Márquez-Linares, M. A., González-Elizondo, S., \& Alvarez-Zagoya, R. (1999). Componentes de la diversidad arbórea en bosques de pino encino de Durango, Méx. Madera y Bosques, 5(2), 67-78. doi: 10.21829/myb.1999.521348

Medrano Meraz, M. D. J., Hernández, F. J., Corral Rivas, S., \& Nájera Luna, J. A. (2017). Diversidad arbórea a diferentes niveles de altitud en la región de El Salto, Durango. Revista Mexicana de Ciencias Forestales, 8(40), 57-68. doi: 10.29298/rmcf.v8i40.36

Mejía-Canales. A., Franco-Maas S., Endara A., A. R., \& Ávila A., V. (2018). Caracterización del sotobosque en bosques densos de pino y oyamel en el Nevado de Toluca, México. Madera y Bosques, 24(3), e2431656. doi: 10.21829/myb.2018.2431656

Monárrez-González, J. C., Pérez-Verdín, G., López-González, C., Márquez-Linares, M. A. y González-Elizondo, M. S. (2018). Efecto del manejo forestal sobre algunos servicios ecosistémicos en los bosques templados de México. Madera y Bosques, 24 (2), e2421569. doi: 10.21829/myb.2018.2421569

Monarrez-Gonzalez, J. C., Gonzalez-Elizondo, M. S., Marquez-Linares, M. A., Gutierrez-Yurrita, P. J., \& Perez-Verdin, G. (2020). Effects of forest management on tree diversity in temperate ecosystem forest in northern Mexico. PLoS ONE, 15(5), e0233292. doi: 10.1371/journal.pone.0233292

Mueller-Dombois, D. \& Ellenberg, H., \&. (1974). Aims and Methods of Vegetation Ecology. New York: John Wiley \& Sons.

Návar-Cháidez, J., \& González Elizondo, M. (2009). Diversidad, estructura y productividad de bosques templados de Durango, México. Polibotánica, (27), 71-87.

Nash, D. L., \& Nee, M., (1984). Verbenaceae. Flora de Veracrur, 41, 1-154.

Ramírez-Santiago, R., Ángeles-Pérez, G., Hernández-de la Rosa, P., Cetina-Alcalá, V. M., Plascencia-Escalante, F. O., \& Clark-Tapia, R. (2019). Efectos del aprovechamiento forestal en la estructura, diversidad y dinámica de rodales mixtos en la Sierra Juárez de Oaxaca, México. Madera y Bosques, 25(3), e2531818. doi: $10.21829 /$ myb.2019.2531818

Ross, M. L., Flanagan, L. B., \& La Roi, G. H. (1986). Seasonal and sucessionl changes in light quality and quantity in the understory of boreal forest ecosystems. Canadian Journal of Botany, 64(11), 2792-2799. doi.org/10.1139/b86-337

Secretaría del Medio Ambiente y Recursos Naturales [Semarnat] (2010). Protección ambiental-especies nativas de México de flora y fauna silvestres. Categorías de riesgo y especificaciones para su inclusión, exclusión o cambio. Lista de especies en riesgo. México; Diario Oficial de la Federación. Recuperado de http://biblioteca.semarnat.gob.mx/janium/Documentos/ Ciga/agenda/DOFsr/DO2454.pdf

Smith, D. M., Larson, B. C., Kelty, M. J., \& Ashton, P. M. S. (1997). The practice of silviculture: applied forest ecology. New York: John Wiley \& Sons, Inc.

Solís Moreno, R., Aguirre Calderón, Ó. A., Treviño Garza, E. J., Jiménez Pérez, J., Jurado Ybarra, E., \& Corral-Rivas, J. (2006). Efecto de dos tratamientos silvícolas en la estructura de ecosistemas 
forestales en Durango, México. Madera y Bosques, 12(2), 49-54. doi: $10.21829 /$ myb.2006.1221242

Stephen, A. H. \& Chen, J. Y. H. (2008). Fire, logging, and overstory affect understory abundance, diversity, and composition in boreal forest. Ecological Monographs, 78(1), 123-140. doi: 10.1890/062140.1

The Plant List (2013). Recuperado de http://www.theplantlist.org

The R Core Team (2019) R: A language and environment for statistical computing, version 3.6.1. Vienna, Austria.

Vásquez-Cortez, V. F., Clark-Tapia, R., Manzano-Méndez, F., GonzálezAdame, G., \& Aguirre-Hidalgo, V. (2018). Estructura, composición y diversidad arbórea y arbustiva en tres condiciones de manejo forestal de Ixtlán de Juárez, Oaxaca. Madera y Bosques, 24(3), 1-13. doi: 10.21829/myb.2018.2431649

Wan Razali, W. M. \& Wan Mohd.Shukri, W. A. (1999). An evaluation of statistical reliability in SMS's pre-felling inventory: the case for confidence and error levels. Journal of Tropical Forest Science. 11(1):11-25. Recuperado de https://www.jstor.org/stable/43582511?seq=1

Wolf, J. H. D. (2005). The response of epiphytes to anthropogenic disturbance of pine-oak forests in the highlands of Chiapas, México. Forest Ecology and Management, 212, 376-393. doi: 10.1016/j.foreco.2005.03.027
Zacarias-Eslava, L. E., Cornejo-Tenorio, G., Cortés-Flores, J., GonzálezCastañeda, N., \& Ibarra-Manríquez, G. (2011). Composición, estructura y diversidad del cerro El Águila, Michoacán, México. Revista Mexicana de Biodiversidad, 82(3), 854-869. doi: 10.22201/ib.20078706e.2011.3.684

Zar, J. H. (2010). Biostatistical analysis. Pearson new international edition (5th ed.). New Jersey, USA: Prentice Hall.

\section{Manuscrito recibido el fecha}

Aceptado el fecha

Publicado el 9 de julio de 2021

Este documento se debe citar como:

Rendón-Pérez, M. A., Hernández-de la Rosa, P., VelázquezMartínez, A., Alcántara-Carbajal, J. L., \& Reyes-Hernández, V. J. (2021). Composición, diversidad y estructura de un bosque manejado del centro de México. Madera y Bosques, 27(1), e2712127. doi: 10.21829/myb.2021.2712127

Madera y Bosques por Instituto de Ecología, A.C. se distribuye bajo una Licencia Creative Commons Atribución-NoComercialCompartirlgual 4.0 Internacional. 